\title{
El comercio exterior y la nueva tendencia del comercio electrónico internacional
}

\section{Foreign trade and the new trend of international electronic commerce}

1 Nubia Elizabeth Casquete Baidal

Universidad de Guayaquil, Guayaquil, Ecuador nubia.casqueteb@ug.edu.ec

2 Omar Gabriel Mejía Flores Investigador E-IDEA OMWIN S.A, Guayaquil, Ecuador omar.mejiaf@estudioidea.org

3 Lissette Fernanda Alvarado Barrera Universidad Ecotec, Samborondón, Ecuador lalvaradob@ecotec.edu.ec https://orcid.org/0000-0002-4683-1606

https://orcid.org/0000-0001-5129-8933

http://orcid.org/0000-0003-2957-9354

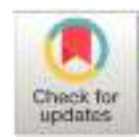

Artículo de Investigación Científica y Tecnológica

Enviado: 11/12/2021

Revisado: 26/12/2021

Aceptado: 28/01/2022

Publicado:05/02/2022

DOI: https://doi.org/10.33262/ap.v4i1.1.180

Casquete Baidal, N. E., Mejía Flores, O. G., \& Alvarado Barrera, L. F. (2022). El comercio exterior y la nueva tendencia del comercio electrónico internacional. AlfaPublicaciones, 4(1.1), 473-484. https://doi.org/10.33262/ap.v4i1.1.180

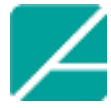

Ciencia

Digital

ALFA PUBLICACIONES, es una Revista Multidisciplinar, Trimestral, que se publicará en soporte electrónico tiene como misión contribuir a la formación de profesionales competentes con visión humanística y crítica que sean capaces de exponer sus resultados investigativos y científicos en la misma medida que se promueva mediante su intervención cambios positivos en la sociedad. https://alfapublicaciones.com

La revista es editada por la Editorial Ciencia Digital (Editorial de prestigio registrada en la Cámara Ecuatoriana de Libro con No de Afiliación 663) www.celibro.org.ec

Esta revista está protegida bajo una licencia Creative Commons Attribution Non Commercial No Derivatives

4.0 International. Copia de la licencia: http://creativecommons.org/licenses/by-nc-nd/4.0/ 


\section{Palabras}

claves:

comercio

exterior, bienes, servicios,

tecnología, comercio electrónico, comercio.

\section{Keywords:}

foreign trade, goods, services, technology, electronic commerce, commerce.

\section{Resumen}

El mundo globalizado afronta constantes cambios a nivel de economías, comercio, finanzas, nuevas tecnologías, etc., que permiten modificar la forma de llevar a cabo todas estas operaciones. El comercio exterior existe desde hace ya mucho tiempo, este se originó para cubrir las necesidades que en un determinado momento no podían ser cubiertos por una región o país, lo cual conllevo a negociar con otros, a través de intercambios que le permitieran obtener aquellos bienes de los cuales carecía. De allí que el comercio exterior, represente una oportunidad de desarrollo y crecimiento para cualquier país, por lo cual se deben adecuar y actualizar las técnicas y procesos para llevarlo a cabo, adoptando todos los cambios que se presenten. En la actualidad aparece el comercio electrónico internacional, el cual permite la comercialización de productos a través de medios tecnológicos como teléfonos e internet, a través de procedimientos muy sencillos y sin tantos protocolos, como los que hay que seguir cuando las naciones se ajustan al comercio exterior.

\section{Abstract}

The globalized world faces constant changes at the level of economies, trade, finance, new technologies, etc., which allow modifying the way all these operations are carried out. Foreign trade has existed for a long time, it originated to cover the needs that at a certain moment could not be covered by a region or country, which led to negotiate with others, through exchanges that allowed them to obtain those goods of which it lacked. Hence, foreign trade represents an opportunity for development and growth for any country, for which the techniques and processes to carry it out must be adapted and updated, adopting all the changes that arise. At present, international electronic commerce appears, which allows the commercialization of products through technological means such as telephones and the Internet, through very simple procedures and without many protocols, such as those that must be followed when nations adjust to foreign trade. 


\section{Introducción}

La necesidad de los países de lograr un crecimiento económico, que le permita mantener una economía estable y les proporcione un desarrollo en todos los ámbitos, les obliga a incursionar en el comercio exterior, dado a que a través de este se abren una gama de opciones, a través de las cuales se pueden obtener, materias primas, bienes, servicios y nuevas tecnologías, con los cuales se puede incrementar el desarrollo económico y social de cualquier país.

Para Aizaga (2021) el comercio exterior es aquella actividad mercantil que se extiende fuera de las fronteras de un país, por lo cual está sujeta a legislaciones, normas, usos, costumbres, tipo de transporte, fuentes de financiamiento, y a las instituciones y convenios internacionales, de los países que se involucraran.

Por otra parte, Pinto \& Rea (2021), el comercio exterior como un intercambio ordinario, de productos a cambio de dinero, con la diferencia que este intercambio atraviesa una frontera.

Según Chávez \& Ponce (2019), el comercio exterior se define como el intercambio de bienes, servicios, tecnología entre varios países, el cual se puede dar de manera unilateral, bilateral o multilateral, los productos a comercializar pueden ser materia prima, productos acabados, finales, intermedios, al igual que el intercambio de conocimientos.

Por ende, se puede resumir que el comercio exterior, es toda aquella actividad mercantil o comercial, que permite el intercambio de bienes, servicios, tecnologías, entre países, los cuales se adhieren al cumplimiento de una serie de normas y legislaciones, y que permite escoger la forma de llevarlo a cabo, a través de convenios que son regulados por instituciones internacionales, responsables de la regulación de esta actividad, así como también la incursión al comercio electrónico, parte fundamental de esta investigación, cuyo objetivo es evaluar las nuevas tendencias del comercio exterior entre ellas el comercio electrónico internacional.

\section{Metodología}

La investigación se llevó a cabo bajo la metodología basada en la investigación documental, a través de la revisión bibliográfica, de documentos de carácter científico, artículos, publicaciones académicas, tendencias en el mercado, medios electrónicos, de diversas índoles, se constata las ideas comúnmente adoptadas por los especialistas, para en un análisis descriptivo y desarrollar el tema de Comercio Exterior y la Nueva Tendencia el comercio electrónico.

El comercio exterior es una práctica desde tiempos muy remotos, la cual ha ido evolucionando a través del tiempo con el fin de mejorar los intercambios comerciales 
entre los países y que hoy en día se enfrenta a los distintos cambios derivados de la globalización y las nuevas tecnologías, como lo es el comercio electrónico.

\section{Resultados y Discusión}

\section{Ventajas del Comercio Exterior}

Ramos (2016), afirma que un país es capaz de fabricar sus propios bienes y servicios, pero también tiene deficiencias de algunos insumos (humanos, naturales, financieros e industriales), lo cual hace necesarios el intercambio con otros países, a través de la exportación (vender) y la importación (comprar), lo cual trae como ventajas:

- $\quad$ Impulsar el bienestar económico y social de una nación

- $\quad$ Estabilización de precios

- $\quad$ Disminución del desempleo

- $\quad$ Aumento de la productividad y competitividad

- Menos riesgos de pérdidas económicas.

Por ende, se puede afirmar que la incursión en el comercio exterior, representa para cualquier país una oportunidad para alcanzar un crecimiento económico, que le permita brindar a sus ciudadanos una mayor estabilidad y bienestar social.

\section{Desventajas del Comercio Exterior}

Chávez \& Ponce (2019), señalan como desventajas:

- $\quad$ Aumento de los impuestos

- Deterioro de mercancías

- Inestabilidades políticas en el país destino

- Falta de interés en la producción nacional

- Comercio ilegal, originando la piratería y contrabando

\section{Aspectos relevantes del Comercio Exterior:}

Para Valencia (2019), la política comercial, representa un factor crítico en la economía de un país y necesario para el ámbito comercial, ya que a través de esta se optimizará y organizará las relaciones comerciales que se puedan implementar con otros países. Dependiendo de los gobiernos están políticas podrían orientarse al libre mercado o en su defecto una proteccionista.

La política comercial cuenta con varios elementos que son: 
- Balanza de Pagos: Según Ramos (2016), es aquella donde se registran todas las transacciones económicas que realizan los residentes de un país con el resto del mundo.

Sus principales componentes son: la cuenta corriente, la cuenta de capital y la cuenta de las reservas oficiales. Estas transacciones se reflejan a través de créditos cuando el caso se trate de pagos recibidos por extranjeros y de débitos cuando se envía pagos a extranjeros.

Dentro de la balanza de pagos se encuentra la balanza comercial: según Valencia (2019), es donde se reflejan y registran todas aquellas importaciones y exportaciones que realiza un país en un tiempo determinado. Se dice que la balanza está en superávit cuando las exportaciones superan a las importaciones, por el contrario, si las exportaciones son menores se dice que hay un déficit.

Por ende, lo ideal sería mantener un mayor volumen en las exportaciones, ya que esto se traduce en una mejor economía.

- Exportaciones: representan todas las mercancías que salen de un país, a través de vías autorizadas y cumpliendo con todos los requisitos aduaneros.

Igualmente, para Aizaga (2021), las exportaciones son aquellas ventas que van más allá de las fronteras arancelarias donde está ubicada la empresa, las cuales generan divisas a los países convirtiéndose en un aporte muy importante y fundamental para el desarrollo de cualquier país.

- Importaciones: Son todas aquellas mercancías que ingresan a un país, las cuales deben cumplir con todas las regulaciones aduaneras del país que recibe.

Por otra parte, Aizaga (2021), mantiene que las importaciones son todas aquellas compras o entradas de productos a un país, fabricados en otro país.

\section{Beneficios del Comercio Exterior:}

Según Flores (2019), entre los beneficios que otorga el comercio exterior están:

\section{En el ámbito macroeconómico:}

Las naciones del mundo se orientan a la especialización productiva, obedeciendo a dos factores:

- La desigualdad de los factores productivos (tierra, trabajo y capital)

- La desigualdad en la constitución tecnológica de los productos, lo cual origina ventajas y desventajas comparativas.

En el ámbito microeconómico: 
Se beneficia el sector exportador y el sector importador.

- Favorece al importador, ya que se puede contar con una gran variedad de productos, los cuales se ponen a disposición del consumidor, con características de calidad y mejor precio.

- Favorece al exportador ya que:

- Puede ampliar su mercado

- Logra reducir o eliminar los desniveles estacionales de la demanda interna del país.

- Logra disminuir o eliminar las caídas de las ventas por los diversos factores que pueden presentarse de forma imprevista.

- Aumenta la producción.

- Cala de una manera más eficiente los costos fijos.

- Mejora en los productos, atendiendo las exigencias que son impuestas por el mercado internacional.

- Opción de optar por apoyos crediticios.

- Mantiene motivados a los integrantes de una empresa.

\section{Los Acuerdos comerciales internacionales - Tipos}

Para Ilquimiche (2018), un acuerdo comercial internacional, son acuerdos que pueden ser bilaterales o multilaterales entre estados, pueden ser llamados convenios o tratados a través de los cuales las naciones se comprometen a cumplir las condiciones que regirán el intercambio y las condiciones establecidas en el.

Tipos de acuerdos comerciales

\section{Según su naturaleza:}

- De cooperación internacional: Se refiere al sistema donde se asocian varios estados con el propósito de lograr objetivos determinados y comunes, los cuales persiguen intereses solidarios, sin que la acción y medidas adoptadas, perturben sus jurisdicciones de estado soberano.

- De integración internacional: Se refiere al sistema a través del cual se unen varios estados, los cuales aceptan no hacer valer unilateralmente sus jurisdicciones y facultado su ejercicio a una autoridad supranacional, en la que se manipulan la fusión de los intereses y que las decisiones se someten al control y procedimientos establecidos. 
Según su tipo de aplicación:

- Acuerdo de primera generación: Son aquellos acuerdos comerciales tradicionales, son simples y divisan aquellos acuerdos de naturaleza comercial que implica la desgravación arancelaria.

- Acuerdo de nueva generación: Es un nuevo tipo de acuerdo comercial que surgió en los años 80. Abarcan no solo el comercio de bienes, sino que también incorpora el comercio de servicios, inversiones, propiedad intelectual y compras estatales, también se incorporan normas de origen, medidas zoosanitarias y medidas antidumpin.

- Tratados de libre comercio (TLC): Son uno de los más utilizados en el continente americano. A través de este convenio dos o más naciones negocian acuerdos generalmente de preferencias arancelarias reciprocas tanto al comercio de bienes como de servicios, también incluye áreas importantes como: inversiones, tecnología, comunicaciones, ambientales, sanitarias, propiedad intelectual, laboral entre otros. A través de estos se otorgan beneficios mutuos para los participantes. Dentro de estos se pueden diferencias tres tipos de tratados: zona de libre comercio, unión aduanera y unión económica. A través de estos tratados se busca la generación de más empleos, la modernización del aparato productivo, la mejora del nivel de vida de la población, la promoción de las inversiones tanto nacionales como extranjeras, la ampliación de los mercados, la promoción del crecimiento económico, el establecimiento de legalidades que regulen las actividades de comercio, garantizas los derechos de todas las personas o empresas que inviertan en el país y promover una competencia justa

\section{El Comercio electrónico internacional}

Según Chávez \& Ponce (2019), definen el comercio electrónico internacional, como aquel que permite internacionalizar todas aquellas actividades que tengan que ver con el intercambio comercial, favoreciendo a las pequeñas y medianas empresas (PYMES), debido a que hoy en día existen diversas plataformas electrónicas con las cuales opera el comercio, y a través de estas se puede mejorar el tipo de información que se proporciona a compradores, proveedores y negociadores, convirtiéndose este canal de comercialización en una mejora para reducir costos y tiempo.

Por otro lado, Zhou (2021), sostiene que el comercio electrónico son los procesos d compra y venta de productos de carácter electrónico, tales como aplicaciones móviles e internet. Incluye las ventas minoristas y compras en línea, así como las transacciones electrónicas.

Asimismo, Zhou (2021), describe la situación del comercio electrónico a nivel global de la siguiente manera: 
El nacimiento del comercio electrónico originado por la revolución tecnológica y la transformación industrial, ha contribuido a las mejoras de la calidad y eficiencia de las operaciones económicas y ha logrado cambiar la producción y el estilo de vida de todos los seres humanos. Igualmente se ha convertido en un pilar fundamental para los ingresos fiscales. Es un comercio que ha logrado una acelerada expansión en todo el mundo.

Igualmente, destaca que el comercio electrónico a apresurado el desarrollo de la economía colaborativa, la cual ha concedido a millones de personas del mundo ser consumidores y al mismo tiempo operadores, así como también, la forma y métodos de conseguir empleo.

Por otra parte, señalo que Estados Unidos ha establecido un entorno propicio para el rápido crecimiento del comercio electrónico, a través de infraestructura y políticas fiscales, lo cual ha originado que se estén formulando leyes y regulaciones para llevarlo a cabo.

De igual forma asegura, que el comercio electrónico internacional, se convertirá en una industria emergente para facilitar las relaciones de compra/venta, traspasando fronteras, así como también las distintas organizaciones internacionales, trabajan en un marco legal multilateral, a fin de establecer un sistema normativo que pueda adaptarse al desarrollo de la economía a través de las redes, al igual que proporcionar un marco normativo de la legislación sobre el comercio electrónico entre países.

\section{Ventajas y Desventajas del Comercio Electrónico}

Para Peña (2019) el comercio electrónico presenta las siguientes ventajas y desventajas:

\section{Ventajas:}

- No hay limitaciones geográficas

- Aumento de clientes online como offline, gracias a la promoción de los productos a través del internet.

- Los costos son menores en comparación al comercio tradicional.

- Mejor forma de mostrar los productos a los empresarios

- Facilidad y rapidez para ubicar los productos

- Se economiza tiempo al momento de hacer las compras

- Se optimiza el tiempo para los negocios y para la atención al cliente.

- Facilita la implementación y desarrollo de las estrategias de marketing.

- Posibilita mostrar más información al comprador sobre los productos y/o servicios.

- Facilita realizar comparaciones de productos, sus características y precio.

\section{Desventajas:}

- $\quad$ Existe mucha competencia, ya que es muy fácil implementar un negocio electrónico. 
- $\quad$ Existe un gran número de personas que no confían en las compras y pagos online.

- Los costos de envío son generalmente muy altos, lo cual es negativo para aquellos negocios pequeños.

- Es más difícil promocionar la tienda online, ya que exige un trabajado personal.

- La seguridad del sitio o página puede generar algunos problemas.

- Se deben estudiar si los productos resultan rentables al venderlos en líneas.

\section{Tipos de comercio electrónico}

Para Osmel (2020), estos tipos se establecen tomando en consideración la relación que existe entre el comprador y el vendedor.

- Business to business (B2B): es aquel comercio de bienes y servicios realizado entre empresas. Encierra el intercambio de datos por internet como medio para la negociación.

- Business to consumer (B2C): es aquel que se realiza entre empresa y los consumidores finales de bienes o servicios, lo cual se puede llevar a cabo a través de catálogos online y concretando la negociación en una plataforma digital.

- Consumer to consumer (C2C): Es aquella que se realiza entre los consumidores finales, sin ningún tipo de intermediación.

- Consumer to Business (C2B): Es aquella que se da entre consumidores y empresas, a través de estas los consumidores demandan un producto por lo cual las empresas ofertan y ofrecen descuentos o mejores planes.

- Administration to business (A2B): es la que se lleva a cabo por la administración y las empresas, donde la administración se encarga de la prestación de servicios a los distintos comercios, como, por ejemplo, auditorias, certificaciones de calidad, promoción exterior, entre otros. Se relacionan las organizaciones empresariales con los gobiernos, a través de medios electrónicos.

- Business to administration (B2A): Es la que se da entre empresas y la administración del estado, a través de la cual las empresas ofrecen sus bienes y servicios a la administración.

- Administration to consumer (A2C): Ocurre entre la administración y los consumidores finales, donde la administración vende sus productos y servicios a los clientes. Facilita las contribuciones entre ciudadanos y gobierno, a fin de realizar trámites online.

En el desarrollo del tema se pudo constatar que el comercio exterior tiene como propósito primordial, asegurar la existencia de ciertas condiciones que garanticen un intercambio de bienes, servicios, tecnologías, talento humano, entre otros, de una forma eficiente y de igualdad para los países que se involucran, garantizando una transparencia en las operaciones realizadas, y que cumplan el objetivo de asegurar la comercialización. 
Para el aseguramiento de esta actividad, existen diversos organismos que regulan estas operaciones, dentro de un marco normativo y legal.

Asimismo, se puede observar que, a través del comercio exterior, se fueron mejorando las condiciones de intercambio comercial entre los países, haciéndolo más flexible permitiendo de esta forma que los países pudieran adquirir aquellos materiales o materias primas que necesitaban para su producción interna.

Sin embargo, en la actualidad la comercialización ha sufrido cambios, a raíz de la aparición del comercio electrónico internacional, el cual ha originado la apertura a la adquisición de muchos rubros a través de este medio, originando en muchos casos el ahorro de dinero y de trámites lentos, al igual que pone a disposición de una gran gama de productos, permitiendo de esta forma que se pueda contar con varias opciones al momento de escoger.

A través del comercio electrónico internacional, las personas y empresas pueden acceder a un variado conjunto de productos disponibles y que pueden ser adquiridos sin límite de tiempo ni ubicación, facilitando de esta forma el proceso de comercialización.

\section{Conclusiones}

- Resulta relevante decir, que, gracias a la globalización y a las nuevas tecnologías, los países pueden incursionar en una comercialización más efectiva y veloz, a través del uso del internet, como principales recursos para el comercio electrónico internacional.

- El nacimiento de la cultura y la educación digital permiten el incremento de acceso a compras y pagos a través de medios digitales, entendiendo por supuesto, tanto los beneficios como los riesgos de compras en línea.

- Para ello los gobiernos trabajan en implementar normas y leyes que regulen este tipo de comercio, con el propósito de brindar seguridad a todo aquel que opte por usar este medio.

- Sin embargo, es importante resaltar, que el comercio electrónico internacional, fomenta el comercio en menor escala, mientras que el comercio exterior, es aquel que permite la comercialización de grandes cantidades de productos, bienes o materias primas que un país requiere bien para suplir sus necesidades o para terminar un producto que luego será comercializado fuera de sus fronteras.

- Ambos tipos de comercios representan para cualquier país un beneficio económico, ya que, a través de ellos, pueden incrementar sus ingresos y contribuir con la economía interna. 


\section{Referencias Bibliográficas}

Aizaga, M. (2021). Plan para la creación de una oficina consultora de comercio exterior y finanzas en la Ciudad de Tabacundo. Otavalo: Trabajo especial de grado de la Universidad de Otavalo para optar al título de Licenciatura en Comercio Exterior y Finanzas.

Chávez, A., \& Ponce, G. (2019). El comercio exterior en el emprendimiento de la producción técnica. Guía de lineamientos básicos de comercio exterior. Guayaquil: Trabajo especial de grado de la Universidad de Guayaquil.

Flores, C. (2019). El comercio exterior en la formación técnica productiva. Ecuador: Trabajo especial de grado de la Universidad de Guayaquil.

Ilquimiche, M. (2018). Acuerdos internacionales comerciales y las exportaciones no tradicionales del Perú años 1990-2016. Trabajo especial de grado de la Universidad del Callao.

Osmel, L. (2020). E-commerce: una visión transcompleja de los negocios internacionales. Agentes de Cambio 3(5), 4-28.

Peña, Y. (2019). Comercio electrónico ventajas y desventajas. Bogotá: Trabajo especial de grado de la Universidad Cooperativa de Colombia.

Pinto, L., \& Rea, B. (2021). Incidencia del comercio exterior en el desarrollo humano en Ecuador en el período 2000-2019. Quito: Trabajo especial de grado de la Universidad Central del Ecuador para optar al título de Economista.

Ramos, A. (2016). Introducción al comercio exterior. Informe.

Valencia, E. (2020). Negocios internacionales vs comercio exterior: Análisis comparativo y tendencias actuales. Ecuador: Trabajo Doctoral de la escuela de Comercio Exterior de PUCESE.

Zhou, C. (2021). El comercio electrónico global y su influencia sobre el desarrollo económico. España: Trabajo especial de grado de la Universidad Politécnica de Cartagena.

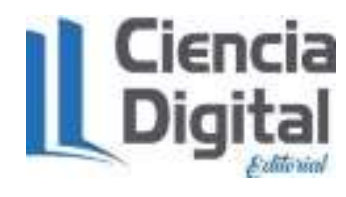


El artículo que se publica es de exclusiva responsabilidad de los autores y no necesariamente reflejan el pensamiento de la Revista Alfa Publicaciones.

\section{Ciencia}

El artículo queda en propiedad de la revista y, por tanto, su publicación parcial y/o total en otro medio tiene que ser autorizado por el director de la Revista Alfa Publicaciones.
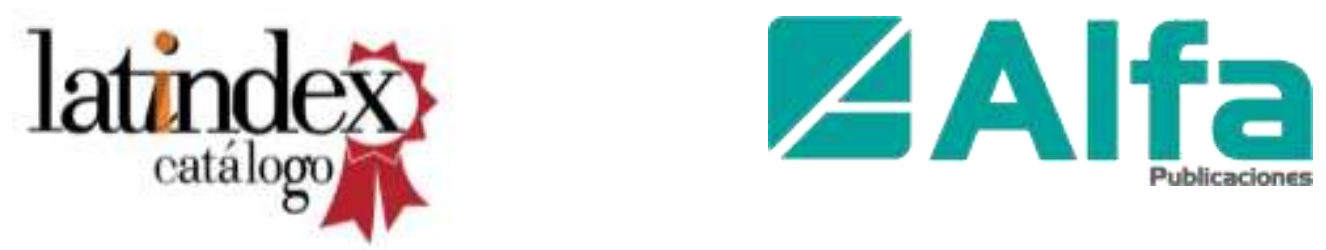

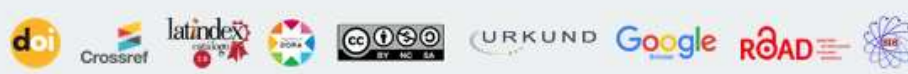
DLatinREV

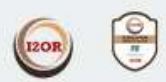

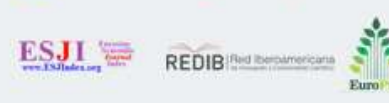

$\underbrace{}_{\text {wizdom.ai }} \mathrm{O}_{\text {OpenAIRE }}^{+}$

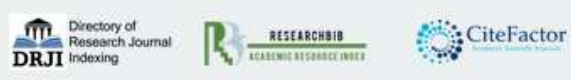

